\title{
Efectos del financiamiento público a la innovación: perspectiva microeconómica a partir de un estudio en pequeñas empresas
}

Effects of public financing for innovation: microeconomic perspective based on a study of small businesses

\author{
María del Pilar Pastor Pérez* \\ Paola Isabel Rodríguez Gutiérrez ${ }^{*}$ \\ Adriana Eugenia Ramos Ávila*
}

Recibido el 13 de noviembre de 2015

Aceptado el 18 de mayo de 2016

Resumen: en teoría, el financiamiento público destinado a incentivar la innovación empresarial está justificado, pero en la práctica existe controversia sobre la evaluación de los impactos derivados del uso de estos recursos. El objetivo de este trabajo es analizar la adicionalidad generada en micro y pequeñas empresas beneficiarias de financiamiento público para la innovación. Para lograrlo se aplicó un cuestionario, y se hizo un análisis descriptivo de las variables adicionalidad de input, de comportamiento y de resultados. Las conclusiones revelan que en la mitad de la muestra existe indicio de adicionalidad en el financiamiento, cuyo resultado más claro es la generación de productos y procesos nuevos, y los efectos en el comportamiento son los más notables. Si bien

* Profesoras de tiempo completo de la Facultad de Contaduría y Administración, Universidad Autónoma de San Luis Potosí. Calle Madero \# 849, colonia Moderna, C. P. 78233, San Luis Potosí, San Luis Potosí, México. Teléfono (444) 834 9917, extensión 105. Correos electrónicos: pilarpastorperez@gmail.com / paolaisabelrg@gmail.com / ady-ramosa@yahoo. com.mx 
el tamaño muestral y el tipo de análisis limitan la generalización de los hallazgos, la evidencia presentada aquí sobre el impacto del financiamiento público en la innovación en economías en desarrollo es relevante, debido a la escasez de trabajos sobre la materia.

Palabras clave: principio de adicionalidad; gestión de la innovación; financiamiento público; evaluación del programa; pequeña empresa.

Abstract: in theory, public financing aimed at encouraging business innovation is justified, but in practice controversy exists over the evaluation of impacts derived from the use of these resources. The aim of this paper is to analyze the additionality generated by micro- and small enterprises benefiting from public financing for innovation. For this purpose, a questionnaire was applied, and a descriptive analysis of the input additionality, performance and results variables was made. The conclusions show that in half of the sample there is evidence of additionality in funding, whose more visible result is the generation of new products and processes, and the effects on performance are the most significant. Although the sample size and the type of analysis limit the generalization of findings, the evidence presented here of the impact of public financing on innovation in developing economies is relevant because of the scarcity of papers on the subject.

Key words: additionality principle; innovation management; public financing; program evaluation; small entreprise.

\section{Introducción}

La innovación es la esencia de las ventajas competitivas sostenibles y del aumento de la productividad de las organizaciones y las econo- 
mías; según la Real Academia Española, ${ }^{1}$ innovación es la “acción y el efecto de innovar", y esto consiste en alterar algo introduciendo novedades. Existe un sinfín de definiciones sobre el término, entre las que se retomó la propuesta del Manual de Oslo, por su amplia difusión y aplicabilidad al estudio: la innovación consiste en la implementación de un bien, servicio o proceso nuevo o significativamente mejorado, o la introducción de un método de comercialización u organización nuevo aplicado a las prácticas de negocio, la organización del trabajo o las relaciones externas (Organisation for Economic Co-operation and Development, OECD, y Eurostat 2005). ${ }^{2}$ Según esta perspectiva, un requisito necesario para que las novedades se consideren una innovación es haber introducido productos en el mercado o utilizado procesos y métodos organizacionales o de mercadotecnia en las corporaciones. La consecuencia de esto es que las empresas son actores fundamentales en la generación de innovaciones, y que no actúan de manera aislada, sino que involucran a otros actores y recursos de su entorno. Esta realidad está reflejada claramente en el enfoque sistémico de la innovación en el que, además de la empresa y sus procesos internos encaminados a innovar, se analiza la repercusión de las relaciones en otras compañías y centros de investigación, y las interacciones con la demanda, la infraestructura disponible y el marco institucional vigente (OECD y Eurostat 2005).

En este marco analítico, la política de innovación juega un papel relevante porque debe ser capaz de reducir las barreras que dificultan el proceso innovador. Entre ellas, las que tienen que ver con la propensión de los empresarios a invertir en actividades de investigación, desarrollo tecnológico e innovación (I+D+i), la cual se ve limitada por las externalidades positivas, los riesgos asociados y la asimetría de información entre innovador e inversionista, que ocasionan problemas de riesgo moral y selección adversa. Estos argumentos se utilizan como justificación teórica de una política pública de fomento a la I+D+i. En la práctica, la discusión sobre cómo articular el financiamiento público es más compleja, en especial cuando se trata de

\footnotetext{
www.rae.es

La definición, en la versión en español, está en la página 56. Se puede consultar en: http:// www.conacyt.gov.py/sites/default/files/detiec/concurso/Manual_de_Oslo.pdf
} 
la evaluación. Algunos aspectos que obstaculizan la valoración de los efectos de una política de promoción de la innovación son:

- El alcance, ya que hay diferencias a la hora de evaluar un programa público, o un proyecto de empresa; también es necesario considerar el alcance territorial de la evaluación.

- La factibilidad de atribuir los resultados innovadores a las medidas evaluadas o a otros factores, como el entorno económico, por ejemplo.

- La dificultad de simular el escenario contrafactual; es decir, los resultados innovadores de no existir la política pública.

- La consideración de los desfases temporales entre las actuaciones de política y el tipo de resultados observables en el corto, mediano o largo plazo (Technopolis Group y Manchester Institute of Innovation Research 2012). ${ }^{3}$

Para incrementar la solidez de las conclusiones relativas a resultados atribuibles a cierta actuación pública, es recomendable utilizar una combinación de métodos cuantitativos y cualitativos (Technopolis Group y Manchester Institute of Innovation Research 2012). Los diseños más empleados son las encuestas a beneficiarios, analizadas a través de las técnicas tanto estadísticas de corte trasversal, como de las econométricas aplicadas a un panel de microdatos suficientemente grande: experimentos o cuasiexperimentos con empresas, grupos de control -firmas innovadoras con características similares al grupo objetivo, que no han recibido financiamiento público-, y análisis de casos de corporaciones beneficiadas.

La mayoría de las evaluaciones publicadas se han realizado en el sector de la manufactura en países desarrollados, y abordan los aspectos cuantitativos asociados a la política pública -Aboal y Garda (2015) ofrecen un repaso amplio sobre el tema-. En este contexto, el trabajo resumido aquí es relevante, porque aporta pruebas del impacto del financiamiento público en la innovación de empresas pequeñas en un país en desarrollo, con hincapié en los efectos cualitativos derivados

3 El texto en español está disponible en: http://ec.europa.eu/regional_policy/sources/docgener/evaluation/pdf/eval2007/innovation_activities/inno_activities_guidance_es.pdf 
de él. El artículo consta de la introducción y tres secciones más; en la segunda se resume la literatura sobre adicionalidad del financiamiento y los programas públicos de apoyo a la innovación en México. Después se explica el método utilizado, en la cuarta se exponen los resultados y al final se incluyen las conclusiones.

\section{Revisión de la literatura}

Adicionalidad del financiamiento público a la innovación

Una de las medidas de política pública más comunes para promover las actividades de $\mathrm{I}+\mathrm{D}+\mathrm{i}$ consiste en otorgar recursos a empresas con potencial innovador. La evaluación de sus efectos debe medir aspectos cuantitativos, como la variación en la inversión empresarial en I+D+i o el lanzamiento de productos nuevos, pero también otros de corte cualitativo, como los cambios en las capacidades de las empresas para seguir generando conocimiento, o en los patrones de cooperación con los actores del sistema de innovación.

Uno de los temas recurrentes gira en torno a la adicionalidad, la cual mide el efecto incentivador de la política pública. En la literatura se distinguen tres tipos básicos de adicionalidad: a) de insumos (o input), calcula el efecto multiplicador del financiamiento público sobre el gasto total en I+D+i de las empresas; b) de resultados (o output), estima los resultados de las actividades de $\mathrm{I}+\mathrm{D}+\mathrm{i}$ que no hubieran sido posibles sin el financiamiento público y c) en el comportamiento, valora las consecuencias sobre el aprendizaje de la organización beneficiada. Esta última, de carácter cualitativo, es posible que se presente a pesar de que no se materialicen las dos primeras, y es de vital importancia en el nivel microeconómico (véase figura 1).

El financiamiento público para actividades privadas de I+D+i se justifica a partir del supuesto de la relación lineal entre la capacidad innovadora y los resultados. Esta premisa aparenta ser simple, pero es compleja de medir (Georghiou et al. 2004). No obstante, se considera que el esfuerzo innovador es una condición necesaria, aunque no siempre sea suficiente para lograr resultados innovadores. Por tanto, el reto del financiamiento público es apoyar a empresas que sin apoyo no hubieran llevado a cabo las actividades de I+D+i, o su alcance hu- 
biera sido menor. A partir de este planteamiento, la adicionalidad de insumo mide si el esfuerzo innovador privado se hubiera realizado sin la ayuda pública, al considerar que se debe observar un efecto complementario y multiplicador, y evitar el desplazamiento de recursos privados (crowding out).

\section{Figura 1}

Adicionalidad del financiamiento público a la innovación

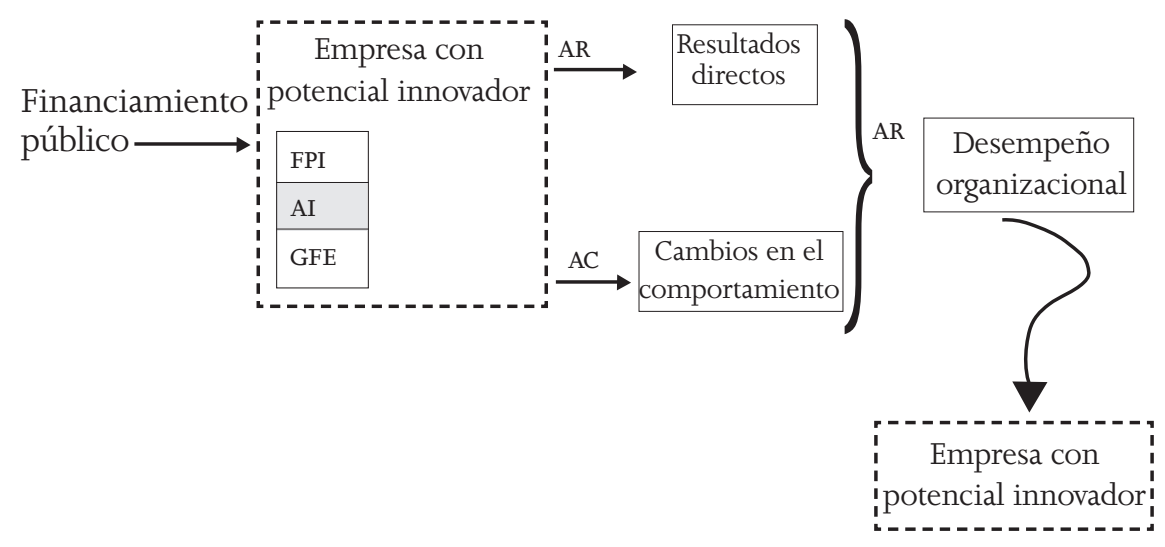

FPI: financiamiento público para la innovación. GFE: gasto inicial financiado por la empresa. AI: adicionalidad de insumos. AR: adicionalidad de resultados. AC: adicionalidad de comportamiento. Fuente: elaboración propia.

La evaluación de la adicionalidad de insumo es la más utilizada, y el indicador empleado más comúnmente para instrumentar la variable insumo es el valor absoluto o la intensidad de la inversión en I+D+i. David et al. (2000) examinaron la literatura y encontraron efectos de sustitución entre la inversión pública y privada, en la tercera parte de los estudios que analizaron. Sin embargo, la evidencia empírica mayoritaria confirma la ausencia del efecto desplazamiento (Almus y Czarnitzki 2003; Duguet 2004; González y Pazó 2008; Aschhoff 2009). 
Lo que se mide en la adicionalidad de resultados es la proporción de los que la empresa no hubiera alcanzado sin la ayuda pública. Es frecuente que, como proxy de los resultados directos de las actividades de I+D+i estén las patentes y los productos innovadores introducidos al mercado. También se utilizan otras variables que miden el desempeño, como la productividad, el empleo o las exportaciones. La detección de estos impactos requiere de tres a cinco años, de lo contrario se corre el riesgo de infravalorarlos. Pero es difícil darle seguimiento a las empresas, lo que complica los análisis econométricos sobre este tipo de adicionalidad.

En lo que respecta a la relación entre el recurso público y el desempeño de la organización la evidencia es menos concluyente que en materia de adicionalidad de insumos; Capron y Van Pottelbergje (1997), Aerts y Czarnitzki (2004) y Hall y Maffiolli (2008) no encontraron relación positiva entre el financiamiento público y la adicionalidad en resultados innovadores, mientras que Bransetter y Sakakibara (2002), Czarnitzki y Hussinger (2004), Czarnitzki et al. (2007) y Czarnitzki et al. (2011) refirieron efectos positivos.

La adicionalidad en el comportamiento existe cuando la corporación beneficiada se comporta de una manera diferente a consecuencia de la ayuda. Los cambios que se miden son en la forma en que las empresas gestionan la I+D+i. Los estudios sugieren que el subsidio acelera la ejecución de los proyectos, expande su escala y alcance y motiva a las compañías a llevar a cabo investigación más desafiante (Lööf y Heshmati 2005; Autio et al. 2008; Clarysse et al. 2009; Hsu et al. 2009). También se pueden producir modificaciones en la estrategia empresarial, y alentar a las empresas a entrar en mercados nuevos, mejorar las habilidades gerenciales, la capacidad de aprendizaje o la colaboración con otros actores (OECD 2006). Cabe señalar que el carácter cualitativo de esta variable aumenta la complejidad de la medición.

En un trabajo sobre empresas innovadoras con y sin financiamiento público en Holanda (2001-2004), Clarysse et al. (2009) se centraron en el proceso de aprendizaje derivado de la ayuda. Estos autores encontraron una correlación fuerte entre la adicionalidad de input y de comportamiento, y explicaron que las empresas dispuestas a cambiar sus métodos de gestión también destinarían recursos para I+D+i. 
Posteriormente, en un estudio sobre el sector textil colombiano y español (2005-2009), Arévalo et al. (2014) retomaron esta idea y definieron a la adicionalidad de comportamiento como la existencia de un mayor esfuerzo innovador de la empresa beneficiaria, ya sea de carácter cuantitativo -inversión de recursos- o cualitativo -cambios en el comportamiento-, gracias a la ayuda. El trabajo confirma la efectividad de las subvenciones a la innovación, mediante la verificación de la adicionalidad de comportamiento -conceptualizada en sentido amplio- $y$ de resultados.

Evidencia de adicionalidad en países en desarrollo

Todavía son escasas las evaluaciones del efecto del financiamiento público a la innovación en los países en desarrollo; en el continente americano se encontraron casos para Argentina, Brasil, Chile, Colombia, México, Panamá y Uruguay; el Banco Interamericano de Desarrollo (BID 2012) resume algunos de ellos. Hay estudios en los que se comparan países: Hall y Maffioli (2008) para Argentina, Brasil, Chile y Panamá, y López-Acevedo y Tan (2010) para Chile, Colombia, México y Perú. A partir de fuentes de datos y métodos de análisis diversos se observa que, en general, el efecto del financiamiento público en la inversión privada en $\mathrm{I}+\mathrm{D}+\mathrm{i}$ es positivo. En los resultados innovadores la evidencia es menos concluyente, y con frecuencia la adicionalidad sobre solicitudes de patentes, productos innovadores, ventas, productividad laboral y empleos, entre otras, no es significativa (véase figura 2).

En México, Calderón-Madrid (2010) evaluó la repercusión del Programa de Estímulos a la Investigación, Desarrollo Tecnológico e Innovación (PEI) para el ejercicio 2009. En relación con la adicionalidad en el gasto, concluyó que el efecto fue positivo, significativo e importante para empresas con entre 10 y 250 trabajadores. En cuanto a la adicionalidad en resultados, el PEI no mejoró la probabilidad que tuvo una empresa de registrar patentes, si además del apoyo del PEI había conseguido crédito fiscal en 2008. Vergara y Heijs (2013) analizaron el efecto del Fondo de Innovación Tecnológica (FIT) en las firmas de la industria química mexicana. Respecto a la adicionalidad financiera, concluyeron que las ayudas del FIT en general tuvieron 


\section{Figura 2}

\section{Estudios sobre adicionalidad en países en desarrollo de América}

\begin{tabular}{|c|c|c|}
\hline País & Estudios & Resultados principales \\
\hline \multirow{4}{*}{ Argentina } & Chudnovsky et al. (2006) & $\begin{array}{l}\text { Adicionalidad en inversión } \mathrm{I}+\mathrm{D}+\mathrm{i} \text { : positiva o sin } \\
\text { evidencia, en función del periodo }\end{array}$ \\
\hline & Binelli y Maffioli (2007) & Adicionalidad en inversión I+D+i \\
\hline & López et al. (2010) & Adicionalidad en inversión I+D+i \\
\hline & Hall y Maffioli (2008) & $\begin{array}{l}\text { Adicionalidad en inversión } \mathrm{I}+\mathrm{D}+\mathrm{i} \\
\text { Ventas de productos nuevos, empleo, exportaciones, } \\
\text { y productividad: efecto no significativo }\end{array}$ \\
\hline \multirow[b]{3}{*}{ Brasil } & De Negri et al. (2006a) & Adicionalidad en inversión I+D+i \\
\hline & De Negri et al. (2006b) & Adicionalidad en inversión I+D+i \\
\hline & Hall y Maffioli (2008) & $\begin{array}{l}\text { Adicionalidad en inversión } \mathrm{I}+\mathrm{D}+\mathrm{i} \\
\text { Patentes, empleo, ventas y productividad: efecto } \\
\text { positivo o no significativo según periodo }\end{array}$ \\
\hline \multirow{4}{*}{ Chile } & Benavente et al. (2007) & Desplazamiento parcial de recursos \\
\hline & Álvarez et al. (2011) & Productividad laboral: efecto positivo \\
\hline & Hall y Maffioli (2008) & $\begin{array}{l}\text { Inversión en } \mathrm{I}+\mathrm{D}+\mathrm{i} \text {, indicadores de resultados } \\
\text { y comportamiento: efecto no significativo } \\
\text { Uso de conocimiento externo: efecto positivo }\end{array}$ \\
\hline & López-Acevedo y Tan (2010) & $\begin{array}{l}\text { Innovación de producto, probabilidad de invertir } \\
\text { en I+D+i, implementación sistemas calidad, } \\
\text { capacitación empleados, empleo: efecto positivo } \\
\text { Ventas y productividad: efecto negativo }\end{array}$ \\
\hline \multirow{3}{*}{ Colombia } & Mercer-Blackman (2009) & Adicionalidad en inversión I+D+i \\
\hline & Crespi et al. (2010) & $\begin{array}{l}\text { Adicionalidad en inversión } \mathrm{I}+\mathrm{D}+\mathrm{i} \\
\text { Introducción de nuevos productos y productividad: } \\
\text { efecto positivo }\end{array}$ \\
\hline & López-Acevedo y Tan (2010) & $\begin{array}{l}\text { Ventas, empleo y salarios: efecto positivo } \\
\text { Exportaciones: efecto no significativo }\end{array}$ \\
\hline \multirow{4}{*}{ México } & Calderón-Madrid (2010) & $\begin{array}{l}\text { Adicionalidad en inversión } \mathrm{I}+\mathrm{D}+\mathrm{i} \\
\text { Patentamiento: no hay evidencia de efecto }\end{array}$ \\
\hline & Vergara y Heijs (2013) & $\begin{array}{l}\text { Adicionalidad en inversión } \mathrm{I}+\mathrm{D}+\mathrm{i} \\
\text { Aprendizaje: efecto positivo }\end{array}$ \\
\hline & Mungaray et al. (2013) & $\begin{array}{l}\text { Vinculación, mejora de las capacidades } \\
\text { del capital humano: efecto positivo }\end{array}$ \\
\hline & López-Acevedo y Tan (2010) & $\begin{array}{l}\text { Ventas y empleo: efecto positivo } \\
\text { Salario: efecto no significativo }\end{array}$ \\
\hline \multirow[b]{2}{*}{ Panamá } & Crespi et al. (2011) & Adicionalidad en inversión I+D+i \\
\hline & Hall y Maffioli (2008) & $\begin{array}{l}\text { Adicionalidad en inversión I+D+i } \\
\text { Productividad, desarrollo y venta de nuevos } \\
\text { productos, acceso a financiamiento, uso de } \\
\text { conocimiento externo: efecto positivo } \\
\text { Capacitación, exportaciones: efecto no significativo }\end{array}$ \\
\hline Uruguay & López y Rossi (2010) & Adicionalidad en inversión I+D+i \\
\hline Perú & López-Acevedo y Tan (2010) & Ganancias y ventas: efecto positivo \\
\hline
\end{tabular}

Fuente: elaboración propia, a partir del BID (2012) y Aboal y Garda (2015). 
consecuencias muy positivas. La adicionalidad de comportamiento es limitada en términos de cooperación y elevada en el aprendizaje. Mungaray et al. (2013) evaluaron el fondo mixto y el PEI (2009) de Baja California. Sus resultados muestran que estas políticas nacionales inciden en el comportamiento de empresas e instituciones de educación superior que, al vincularse a través de los fondos, incrementan su competitividad.

Programas públicos de apoyo a la innovación en México

La proporción del gasto en investigación científica y desarrollo experimental (GIDE) con respecto al producto interno bruto (PIB) es un indicador muy utilizado a escala internacional, para comparar la inversión que cada país destina a proyectos de investigación científica y desarrollo experimental. En conjunto, los países de la Organización para la Cooperación y Desarrollo Económicos (OCDE) invierten 2.3 por ciento del PIB - de 2.14 del año 2000, se incrementó a 2.37 en 2014-. En México, esta variable era de 0.33 por ciento en el año 2000, y en 2011 llegó a 0.43, y la estimación para 2014 fue de 0.54 (OECD 2016). En términos absolutos, en 2013, el GIDE ascendió a 80 812 millones de pesos (Consejo Nacional de Ciencia y Tecnología, CONACYT 2015).

Una característica trascendental de esta inversión es la fuente de financiamiento. En los países más desarrollados, la aportación del sector privado es sustancialmente mayor que la del público. En 2013, en la OCDE esta distribución fue de 60.50 y 28.35 por ciento respectivamente; mientras que en México los valores en 2011 fueron de 36.76 y 59.62, y para 2013 de 22.16 y 74.49. En México, la participación del sector privado en el financiamiento de estas actividades se ha ido incrementando desde el año 2000, cuando el indicador se ubicó en 29.52 por ciento; no obstante, se observa un nuevo retroceso desde 2009 (OCDE 2016). Casi todo el financiamiento público procede de los recursos del gobierno federal, por ejemplo, en 2012 fue de 98.5 por ciento; el monto que aportan los gobiernos estatales y municipales es residual (CONACYT 2014).

Otro indicador utilizado en México es el gasto nacional en ciencia, tecnología e innovación (GNCTI), que además incluye la inversión en 
educación de posgrado, los servicios científicos y tecnológicos y la innovación. En 2012, el GNCTI fue de 114474.1 millones de pesos, 0.74 por ciento del PIB. De este monto, 58.3 se destinó a la investigación científica y desarrollo experimental, 20.6 a la educación de posgrado, 19.1 a servicios científicos y tecnológicos y 2.0 a innovación. Este último concepto se contabiliza de forma separada desde 2007, y contempla el presupuesto asignado para estimular la innovación en empresas a través de programas que operan el CONACYT y la Secretaría de Economía (CONACYT 2014).

Entre dichos programas públicos, por los recursos destinados destaca el PEI que, de 2009 a 2015, otorgó apoyos por 18663 millones de pesos, los cuales se conjuntaron con una inversión privada de 21 756 millones. El total se repartió en 4613 proyectos innovadores y 3554 empresas, de las cuales 66.15 por ciento fue de tamaño micro o pequeño. La distribución regional revela que Nuevo León, Jalisco, Distrito Federal y Estado de México recibieron más recursos públicos -36.94 por ciento de la inversión del periodo y 34.27 de los proyectos beneficiados-. Coahuila, Puebla, Guanajuato, Chihuahua y Sonora acumularon 22.70 por ciento de los recursos y 24.82 de los proyectos. Los sectores más apoyados fueron tecnologías de la información, automotriz, alimentos, agroindustrial, química y biotecnología -más de mil millones de pesos para cada uno- (CONACYT 2015).

\section{Método}

El diseño de esta investigación fue no experimental de alcance descriptivo, cuyo objetivo fue analizar la adicionalidad generada en empresas beneficiadas con financiamiento público para la innovación. La adicionalidad se concibe como la mejora en el esfuerzo innovador, cuantitativo y cualitativo, derivado de las ayudas públicas que reciben las empresas (Clarysse et al. 2009; Arévalo et al. 2014). En este sentido, se incluye la adicionalidad de input y de comportamiento y se relacionan con los resultados.

La evaluación se llevó a cabo desde la perspectiva microeconómica, se centró en el beneficiario, no en el proyecto. Se estudiaron micro y pequeñas empresas con no más de 50 trabajadores, que recibieron 
financiamiento público para la innovación. Se seleccionaron como población objeto de estudio porque son las que tienen menores capacidades de innovación y mayores dificultades para beneficiarse de la ayuda pública (Clarysse et al. 2009; Vila et al. 2010). Además, en gran parte de las organizaciones más pequeñas el apoyo llega a ser vital para que se embarquen o no en un proyecto innovador. Cuando Segura et al. (2014) evaluaron el ambiente innovador en San Luis Potosí, afirmaron que los obstáculos para la innovación varían en función del tamaño de la empresa; para las grandes, la organización es el más importante, y en las micro, pequeñas y medianas destaca el financiamiento y la recuperación de la inversión.

Las empresas micro y pequeñas seleccionadas fueron beneficiarias de las convocatorias del PEI en San Luis Potosí, de 2009 a 2012. De ellas, 15 cumplieron con estos criterios -73.3 por ciento ubicadas en el área conurbada de San Luis Potosí y Soledad de Graciano Sánchez, 20 en otros municipios del estado y una en León, Guanajuato-. El objetivo planteado fue elaborar un censo, por lo que se intentó contactarlas a todas. Sin embargo, sólo se obtuvo información de diez; de las cinco restantes, una ya no operaba, otra no se localizó y tres no accedieron a responder el cuestionario.

En cuanto a la justificación del caso de estudio, su elección se sustentó en dos argumentos: a) el PEI es el instrumento principal de la política pública mexicana, para potenciar la inversión privada en I+D + i y b) analizar lo que sucede en San Luis Potosí es relevante, porque permite explorar qué ocurre en los estados que no son líderes en captación de recursos públicos de este fondo, pero que tienen actividad suficiente para ser susceptibles de análisis. En este sentido, entre 2009 y 2015, según datos publicados por el CONACYT (2015), San Luis Potosí se ubicó en la mitad superior de la clasificación, por número de proyectos apoyados y recursos captados, pero no fue una de las entidades dominantes. Coahuila, Chihuahua y Sonora estaban en una situación similar.

Las variables del estudio fueron la adicionalidad de insumo, de resultados y en el comportamiento (véase figura 3). A partir de los cuestionarios utilizados por Clarysse et al. (2009) y Hsu et al. (2009) se diseñó uno para recolectar la información, integrado por 16 preguntas cerradas de opción múltiple o con una escala tipo Likert, y en 
la mayoría se eliminó la opción de respuesta neutral, para forzar a los encuestados a expresar acuerdo o desacuerdo con lo establecido en el enunciado.

\section{Figura 3}

Variables de estudio

\begin{tabular}{|c|c|}
\hline Variables & Criterios de medición \\
\hline $\begin{array}{l}\text { Adicionalidad } \\
\text { de insumo }\end{array}$ & Efecto complementario en el presupuesto en $\mathrm{I}+\mathrm{D}+\mathrm{i}$ \\
\hline \multirow{21}{*}{$\begin{array}{l}\text { Adicionalidad en el } \\
\text { comportamiento }\end{array}$} & Estrategia innovación \\
\hline & Escala del proyecto ampliada \\
\hline & Objetivos del proyecto ampliados \\
\hline & Ejecución acelerada del proyecto \\
\hline & Involucramiento en investigación de mayor riesgo \\
\hline & Aumento de actividades I+D+i \\
\hline & Formalización del proceso de innovación \\
\hline & Mayor compromiso directivo con la innovación \\
\hline & $\begin{array}{l}\text { Desarrollo de líneas de investigación en paralelo en lugar } \\
\text { de en orden secuencial }\end{array}$ \\
\hline & Desarrollo de una trayectoria de investigación no exitosa \\
\hline & Mejor gestión de las capacidades de innovación \\
\hline & Desarrollo de experiencia en gestión de proyectos \\
\hline & Desarrollo de experiencia tecnológica \\
\hline & Colaboración \\
\hline & Colaboración externa prolongada o nueva \\
\hline & Otros impactos \\
\hline & Cambio en la estrategia de negocio \\
\hline & Menor uso de materiales y consumo de energía \\
\hline & Actualización de recursos humanos \\
\hline & $\begin{array}{l}\text { En la empresa se utilizan habilidades desarrolladas } \\
\text { por las personas involucradas en el proyecto }\end{array}$ \\
\hline & $\begin{array}{l}\text { Actualización de equipo y tecnología con consecuencias } \\
\text { positivas para otros proyectos }\end{array}$ \\
\hline \multirow{7}{*}{$\begin{array}{l}\text { Adicionalidad de } \\
\text { resultados }\end{array}$} & Productos o procesos nuevos o mejorados \\
\hline & Mayor cuota de mercado \\
\hline & Mayor rentabilidad \\
\hline & Incremento en competitividad \\
\hline & Potencial futuro de innovación \\
\hline & Patentes nuevas u otras figuras de protección industrial \\
\hline & Reducción de costos \\
\hline
\end{tabular}

Fuente: elaboración propia a partir de Clarysse et al. (2009) y Hsu et al. (2009). 
Las preguntas del cuestionario versaron sobre los temas siguientes: datos generales -actividad de la organización, año en el que se recibió el apoyo y número de proyectos-; tipo de innovación generada -producto o proceso-; grado de la novedad; introducción en el mercado o implementación del resultado del proyecto; impacto de la introducción o implementación en la oferta, cuota de mercado y competitividad de la empresa; actividad innovadora más allá de los proyectos beneficiados por el programa público -durante y después del apoyado por el PEI-; estrategia de propiedad intelectual; ejecución o no del proyecto sin el recurso público recibido y alternativas financieras probables; valoración del efecto del financiamiento público en la velocidad, escala, colaboración y alcance del proyecto comparado con el escenario de no haber sido beneficiarios; impacto del proyecto en la estrategia de innovación de la empresa -riesgos asumidos, actividades innovadoras de largo plazo, líneas nuevas de investigación-; impacto en la colaboración con otras organizaciones; impacto en el desempeño de la organización -rentabilidad, costos, empleos, exportaciones, consumo de energía- y otros, como actualización de recursos humanos, capacidad de gestión de la innovación, aprovechamiento de habilidades desarrolladas y formalización del proceso de innovación.

El levantamiento de la información se llevó a cabo en el segundo semestre de 2014. Para seleccionar a los informantes clave, se solicitó a las empresas que aceptaron participar, que la persona que respondiera el cuestionario debía haber estado involucrada en el proyecto apoyado por el PEI, y conocer su desarrollo y efecto en la organización, como los líderes de los proyectos o los gerentes.

La limitante del estudio es que, como el análisis fue descriptivo, no se pudo atribuir la causalidad de los resultados obtenidos a la ayuda pública. Sin embargo, a partir de la base del argumento contrafactual, cabe reflexionar sobre la inferencia de causalidad. Desde la perspectiva empírica, el problema es que la situación contrafactual no se puede observar, pero en el cuestionario hubo preguntas en las que se solicitó comparar la ejecución del proyecto con la situación hipotética de no haber recibido financiamiento público. Dado el perfil de los informantes clave, es relevante la comparación con el escenario alternativo y, a partir de esto, su percepción de los efectos del apoyo público en la organización. 


\section{Resultados}

Caracterización de la muestra

La muestra se integró con la misma cantidad de empresas micro (entre 0 y 10 empleados) y pequeñas (11 a 50), pertenecientes a los sectores de alimentos, madera y papel, aeroespacial, química, equipo de medición y control, biotecnología y servicios en el manejo de materiales en la industria. Una de ellas fue beneficiaria en 2009, dos en 2010, cuatro en 2011 y tres en 2012. Para 90 por ciento de los casos analizados, el financiamiento del PEI fue el primer apoyo público recibido para innovar (véase figura 4).

\section{Figura 4}

\section{Perfil de la muestra}

\begin{tabular}{|l|c|}
\hline $\begin{array}{l}\text { Actividad } \\
\text { productiva }\end{array}$ & $\begin{array}{c}\text { Alimentos, madera y papel, aeroespacial, química, equipo de } \\
\text { medición y control, biotecnología y servicios para la industria }\end{array}$ \\
\hline Tamaño & $\begin{array}{c}\text { Microempresas: } 50 \% \\
\text { Pequeñas: } 50 \%\end{array}$ \\
\hline & $(\%)$ \\
Año del apoyo & $2009: 10$ \\
& $2010: 20$ \\
& $2011: 40$ \\
\hline Cantidad de apoyos & $2012: 30$ \\
públicos recibidos & Uno: $90 \%$ \\
\hline
\end{tabular}

Fuente: elaboración propia.

Adicionalidad de insumo

En cuanto a la repercusión del programa público en el financiamiento de la $\mathrm{I}+\mathrm{D}+\mathrm{i}$, las empresas estudiadas reconocieron tres escenarios, de no haber recibido apoyo para el proyecto: a) 40 por ciento no lo hubiera llevado a cabo. Esto indica un efecto multiplicador positivo de la ayuda pública, ya que las beneficiarias del PEI se obligan a destinar recursos concurrentes para la innovación; b) 40 por ciento lo hubie- 
ra hecho con presupuesto menor: una empresa no hubiera asignado otro recurso en lugar del financiamiento, lo que señala un efecto multiplicador positivo de la ayuda, y tres de ellas afirmaron que hubieran destinado más recursos internos o externos de haber sido necesario. Esto es un indicio del desplazamiento de recursos privados provocado por el financiamiento público y c) 20 por ciento aseguró que hubiera desarrollado el proyecto con el mismo presupuesto, con recursos internos, lo que también es una señal del efecto desplazamiento.

A pesar de lo anterior, las empresas afirman estar de acuerdo en que desde que recibieron el financiamiento han aumentado el presupuesto destinado a I+D+i -sólo una estuvo en desacuerdo con esto-. Además, el apoyo tuvo una influencia positiva en la capacidad de atraer recursos adicionales, y en la participación de las empresas en otras convocatorias de financiamiento público a la innovación -80 y 60 por ciento de los casos respectivamente-

Adicionalidad de resultados

En lo concerniente a los resultados directos, la mayoría de las innovaciones derivadas de los proyectos que recibieron el financiamiento público son de producto:

- De los beneficiarios, 60 por ciento desarrolló un producto nuevo o mejoró alguno; 20 un proceso de producción, y 20 innovó en producto y proceso.

- De las ocho empresas que innovaron en producto, la mitad afirmó que se trataba de uno nuevo, y el resto reconoció que mejoró uno ya existente.

- De las empresas que innovaron en producto, 63 por ciento afirmó que cuando se levantó la encuesta sus productos nuevos ya se vendían en el mercado. Tres respondieron que todavía no habían incursionado en el mercado. Estos tres productos no deberían ser calificados como innovaciones, si se considera que sólo lo son en la medida en que llegan al mercado.

- Una empresa solicitó por primera vez una patente y un modelo de utilidad en México, y una patente en el extranjero; otra una patente en México y en el extranjero. Estos hechos también son muestra 
de adicionalidad de resultados, con notable importancia desde la perspectiva de los derechos de propiedad intelectual.

- En cuanto a los procesos, dos empresas los catalogan como nuevos, y las otras dos como una mejora de un proceso ya existente; 75 por ciento reconoció que el efecto de éste fue grande, y el resto que fue limitado.

Con respecto a los cuatro proyectos que se hubieran cancelado de no haber recibido la ayuda pública, cabe discutir si los resultados derivados se pueden considerar como adicionalidad. En principio, la respuesta es afirmativa, pero en sentido estricto el concepto de adicionalidad compara la situación en la que existe un proyecto de innovación con financiamiento público con la ejecución de éste sin el apoyo.

En términos del desempeño empresarial, para 60 por ciento de la muestra, el proyecto dio lugar a un aumento de la rentabilidad y sólo para 30 permitió reducir costos. Para 50 por ciento, el efecto del producto o proceso innovador en la cuota de mercado fue positivo, $y$ para el resto no hubo; para 66.6 el efecto en la competitividad de la empresa fue positivo y en los demás no se presentó; 83.3 por ciento reconoció que el efecto en el potencial de innovación fue positivo y para el resto fue nulo.

\section{Adicionalidad en el comportamiento}

Los cambios en el comportamiento, referidos por los encuestados, son importantes y a continuación se exponen agrupados en tres categorías: estrategia de innovación, colaboración y otros impactos. En cuanto a la relevancia de la primera, para las empresas de menor tamaño -menos propensas a innovar-, destaca el aumento de las actividades de I+D+i. Esto se concluye porque tras la ayuda pública aumentó el número de las que las realizan -durante el año del proyecto apoyado, sólo 20 por ciento de las firmas estaba llevando a cabo uno de I+D+i, mientras que 50 de las encuestadas afirmó que había tenido otros innovadores después de la recepción de los recursos-. Además, 80 por ciento está de acuerdo en que el financiamiento fue útil para convencer a la gerencia de ejecutar el proyecto de innovación, y en que desde entonces se comprometen con regularidad a empren- 
derlos; también 70 por ciento presta más atención a las actividades de $\mathrm{I}+\mathrm{D}+\mathrm{i}$.

Lo anterior es coherente con el hecho de que en la mayoría de las empresas las experiencias tecnológicas, en gestión de proyectos y en colaboración, derivadas del proyecto, eran o iban a ser utilizadas en otros de I+D+i. Además, el proyecto aumentó sus capacidades de gestión de la innovación, y dio lugar a un proceso más formal. El financiamiento hizo que la mayor parte de las firmas se comprometieran en investigación de mayor riesgo y desarrollaran líneas de investigación en paralelo, en lugar de en orden secuencial; sólo 20 por ciento mencionó que la trayectoria de investigación no resultó exitosa.

Los aspectos negativos en términos de la estrategia de innovación se refieren al efecto del financiamiento en la celeridad, escala y los objetivos del proyecto realizado. En la mayoría de los casos afirman que éste fue nulo o negativo, lo que da pie a pensar que en estos tres aspectos no existe adicionalidad de comportamiento. Esta percepción, opuesta a lo que sugiere la literatura empírica, podría explicarse debido a la experiencia relativa a la burocracia del programa de financiamiento. Segura et al. (2014) aseveran, a partir de sus resultados sobre el ambiente innovador en San Luis Potosí, que los aspectos mejorables de los programas de apoyo a la innovación más mencionados por los encuestados son los tiempos de proceso para obtener financiamiento, la difusión de los programas y la complejidad de las reglas de operación.

En el caso de la colaboración, la mayor parte manifestó su conformidad en que la repercusión del proyecto fue positiva, porque aumentó la capacidad de establecer contactos con universidades, centros públicos de investigación y otras empresas, y porque han extendido la colaboración con los socios más allá de las exigencias del proyecto financiado.

También se observó que los efectos del equipo adquirido y la tecnología desarrollada fueron positivos para otros proyectos. Todas las firmas reconocieron que gracias al proyecto se actualizó el recurso humano, y para 90 por ciento las habilidades desarrolladas se siguen utilizando. Entre los aspectos en los que el efecto general no fue positivo destaca que la mayoría de los proyectos no provocaron cambios en la estrategia de negocios -contrario a lo que sugiere la OECD 


\section{Figura 5}

\section{Resumen de resultados}

\begin{tabular}{|c|c|}
\hline Variables & Criterios de medición (efecto) \\
\hline $\begin{array}{l}\text { Adicionalidad } \\
\text { de insumo }\end{array}$ & Efecto complementario en el presupuesto en $\mathrm{I}+\mathrm{D}+\mathrm{i}(+)$ \\
\hline \multirow{21}{*}{$\begin{array}{l}\text { Adicionalidad en } \\
\text { el comportamiento }\end{array}$} & Estrategia innovación \\
\hline & Escala del proyecto ampliada (-) \\
\hline & Objetivos del proyecto ampliados (-) \\
\hline & Ejecución acelerada del proyecto (-) \\
\hline & Involucramiento en investigación de mayor riesgo $(+)$ \\
\hline & Aumento de actividades I+D+i (+) \\
\hline & Formalización del proceso de innovación $(+)$ \\
\hline & Mayor compromiso directivo con la innovación (+) \\
\hline & $\begin{array}{l}\text { Desarrollo de líneas de investigación en paralelo, } \\
\text { en lugar de en orden secuencial }(+)\end{array}$ \\
\hline & Desarrollo de una trayectoria de investigación no exitosa (-) \\
\hline & Mejor gestión de las capacidades de innovación (+) \\
\hline & Desarrollo de experiencia en gestión de proyectos $(+)$ \\
\hline & Desarrollo de experiencia tecnológica $(+)$ \\
\hline & Colaboración \\
\hline & Colaboración externa prolongada o nueva $(+)$ \\
\hline & Otros impactos \\
\hline & Cambio en la estrategia de negocio (s.e.) \\
\hline & Menor uso de materiales y consumo de energía (s.e.) \\
\hline & Actualización de recursos humanos $(+)$ \\
\hline & $\begin{array}{l}\text { En la empresa se utilizan habilidades desarrolladas por las } \\
\text { personas involucradas en el proyecto }(+)\end{array}$ \\
\hline & $\begin{array}{l}\text { Actualización de equipo y tecnología } \\
\text { con consecuencias positivas para otros proyectos }(+)\end{array}$ \\
\hline \multirow{7}{*}{$\begin{array}{l}\text { Adicionalidad de } \\
\text { resultados }\end{array}$} & Productos o procesos nuevos o mejorados (+) \\
\hline & Cuota de mercado (s.e.) \\
\hline & Rentabilidad (+) \\
\hline & Incremento en competitividad (+) \\
\hline & Potencial de innovación (+) \\
\hline & Patentes nuevas u otras figuras de protección industrial (s.e.) \\
\hline & Reducción de costos (s.e.) \\
\hline
\end{tabular}

(+): efecto positivo del financiamiento público en la innovación de la empresa.

(-): efecto negativo del financiamiento en el proyecto de innovación.

(s.e.): sin efecto, porque no hay efecto positivo mayoritario (más de 50 por ciento de la muestra).

Fuente: elaboración propia. 
(2006)-, ni redujeron el consumo de energía o materiales, con beneficios positivos para la sustentabilidad -70 y 60 por ciento de la muestra respectivamente-.

A pesar de la existencia de percepciones negativas, la perspectiva general que se deduce de las respuestas es que las empresas reportan experiencias positivas, derivadas de los proyectos innovadores para los que recibieron el apoyo público (véase figura 5).

\section{Conclusiones}

Los impactos de la política pública de financiamiento a la innovación en México están poco explorados a nivel macro y microeconómico. Aunque los programas existentes son analizados por diferentes instancias, no hay indicadores sistemáticos y públicos de impacto y de análisis costo-beneficio. También es importante el seguimiento de las empresas más allá del cierre de los compromisos derivados de la participación en un programa público, lo que permitiría identificar efectos, de mediano y largo plazo, en la organización beneficiaria y en la economía.

El objetivo del estudio fue analizar la adicionalidad generada en empresas beneficiadas con financiamiento público para la innovación. Por su diseño metodológico, no es posible calificar el impacto producido por dicha ayuda, pero se presenta evidencia de su efectividad. Entre las conclusiones principales, destaca que 90 por ciento de las compañías encuestadas relató estar de acuerdo en que el financiamiento público detonó o incrementó las actividades de investigación, desarrollo tecnológico e innovación. Además, hay muestra clara de adicionalidad de financiamiento a la I+D+i en 50 por ciento de los casos. Este panorama es congruente con la literatura empírica.

En relación con la adicionalidad de resultados, 70 por ciento de las empresas afirmó que el producto nuevo ya se había introducido en el mercado o que el proceso ya se había implementado. En términos de desempeño organizacional, también en la mayoría de los estudios las consecuencias son menos concluyentes, pero sobresale el efecto que reconocen los beneficiarios en términos de potencial de innovación. 
La mayoría de las percepciones sobre el impacto del financiamiento público en el comportamiento fueron positivas y, dado el planteamiento de las preguntas del cuestionario, es factible atribuir adicionalidad de comportamiento. Los efectos positivos que se deducen del relato general de las respuestas de las organizaciones estudiadas sugieren que las beneficiarias tienen problemas de apropiación o dificultades financieras, o ambos, pero que a pesar de su tamaño, cuentan con potencial para desarrollar sus capacidades y ser innovadoras. Por esto, es importante que las firmas hayan reconocido los efectos positivos sobre el recurso humano con el que cuentan, la capacidad de gestión de la innovación, la experiencia tecnológica y la colaboración con otros actores.

La radiografía presentada permite observar lo que sucede en empresas pequeñas de un país emergente, para las que la innovación ha sido tradicionalmente un reto mayúsculo. Se advierte que el recurso público se materializa en cambios organizacionales que los líderes perciben con claridad, y esto es fundamental para que se produzcan mejoras en su trayectoria. Si bien el impacto regional es limitado, porque los recursos públicos destinados a promover la innovación en México son marginales en relación con el tamaño de la economía, los hallazgos refuerzan la idea de la bondad de incrementar los apoyos a este tipo de empresas -Mouqué (2012) plantea que éstos son más efectivos para las pequeñas y medianas, que para las grandes-.

Por lo tanto, el diseño de la política debe considerar las particularidades locales y regionales, porque así mejorará su impacto. Por esto, es necesario profundizar en el estudio de las consecuencias de las medidas de financiamiento que se están instrumentando. Además de los análisis a nivel macro, como los estudios económico-financieros de los programas que existen en México, es vital el microeconómico en el que se evalúen los efectos del recurso público en términos de adicionalidad. Para esto se precisa incrementar el tamaño de las muestras estudiadas y recolectar información de corte longitudinal, utilizar técnicas econométricas de análisis, y considerar grupos de control que permitan comparar empresas innovadoras que reciben o no apoyo público para llevar a cabo actividades de I+D+i. 


\section{Bibliografía}

Aboal, Diego y Paula Garda. 2015. ¿La financiación pública estimula la innovación y la productividad? Una evaluación de impacto. Revista CEPAL 115: 45-70.

Aerts, Kris y Dirk Czarnitzki. 2004. Using innovation survey data to evaluate R\&D policy: the case of Belgium. ZEW discussion papers 04-55. Bélgica: Center for European Economic Research.

Almus, Matthias y Dirk Czarnitzki. 2003. The effects of public R\&D subsidies on firms' innovation activities: the case of Eastern Germany. Journal of Business and Economic Statistics 21 (2): 226-236.

Álvarez, Roberto, José Miguel Benavente, Rolando Campusano y Conrado Cuevas. 2011. Employment, firm size and innovation in Chile: the microeconometric evidence. Nota técnica 319. Washington: BID.

Arévalo, Raquel T., Francisco F. López y Dora P. Espinal. 2014. Evidencia sobre adicionalidad de las ayudas públicas a la innovación empresarial. Caso del sector textil-confección. Ponencia presentada en el XXVIII AEDEM annual meeting, Trujillo, España.

Aschhoff, Birgit. 2009. The effect of subsidies on R\&D investment and success: do subsidy history and size matter? Discussion papers ZEW 09-032. Alemania: Zentrum für Europäische Wirtschaftsforschung.

Autio, Erkko, Sammi Kanninen y Robin Gustafsson. 2008. First and second-order additionally and learning outcomes in collaborative R\&D programs. Research Policy 37 (1): 59-76.

Benavente, José, Gustavo Crespi y Alessandro Maffioli. 2007. Public support to firm level innovation: an evaluation of the FONTEC program. Documento de trabajo ove 05/07. Washington: BID. 
BID. 2012. Las instituciones fiscales del mañana. Washington: BID.

Binelli, Chiara y Alessandro Maffioli. 2007. A micro-econometric analysis of public support to private R\&D in Argentina. International Review of Applied Economics 21 (3): 339-359.

Bransetter, Lee y Mariko Sakakibara. 2002. When do research consortia work well and why? Evidence from Japanese panel data. American Economic Review 92: 143-159.

Calderón-Madrid, Ángel. 2010. A microeconometric analysis of the impact of Mexico's R\&D tax credit program on private R\&D expenditure. El Colegio de México. Documento inédito.

Capron, Henri y Bruno van Pottelsberghe. 1997. Public support to business R\&D: a survey and some new quantitative evidence. En Policy evaluation in innovation and technology: towards best practices, compilado por OECD, 171-187. París: OECD.

Chudnovsky, Daniel, Andres López, Martin Rossi y Diego Ubfal. 2006. Evaluating a program of public funding of private innovation activities. An econometric study of FONTAR in Argentina. Documento de trabajo OvE 16/06. Washington: BID.

Clarysse, Bart, Mike Wright y Philippe Mustar. 2009. Behavioural additionality of R\&D subsidies: a learning perspective. Research Policy 38 (10): 1517-1533.

CONACYT. 2015. Programa de Estímulos a la Investigación, Desarrollo Tecnológico e Innovación. Convocatoria 2016. http://www. conacyt.mx/index.php/el-conacyt/convocatorias-y-resultadosconacyt/convocatorias-programa-de-estimulos-a-la-innovacion/ convocatoria-2016/10245-informacion-basica-pei-2016/file (13 de abril de 2016).

CONACYT. 2014. Programa Especial de Ciencia, Tecnología e Innovación 2014-2018. Diario Oficial de la Federación. 30 de julio. México: Secretaría de Gobernación. 
Crespi, Gustavo, Galileo Solís y Ezequiel Tacsir. 2011. Evaluación del impacto de corto plazo de la Secretaría Nacional de Ciencia, Tecnología e Innovación en la innovación de las empresas panameñas. Nota técnica 263. Washington: BID.

Crespi, Gustavo, Alessandro Maffioli y Marcela Meléndez. 2010. Public support to innovation. The Colombian COLCIENCIAS' experience. Nota técnica 264. Washington: BID.

Czarnitzki, Dirk, Petr Hanel y Julio Miguel Rosa. 2011. Evaluating the impact of R\&D tax credits on innovation: a microeconometric study on Canadian firms. Research Policy 40 (2): 217-229.

Czarnitzki, Dirk, Bernd Ebersberger y Andreas Fier. 2007. The relationship between R\&D collaboration, subsides and R\&D performance: empirical evidence from Finland and Germany. Journal of Applied Econometrics 22: 1347-1366.

Czarnitzki, Dirk y Katrin Hussinger. 2004. The link between R\&D subsidies, R\&D spending and technological performance. ZEW discussion papers 04-56. Bélgica: Center for European Economic Research.

David, Paul A., Bronwyn H. Hall y Andrew A. Toole. 2000. Is public $R \& D$ a complement or substitute for private R\&D? A review of the econometric evidence. Research Policy 29: 497-529.

De Negri Joáo A., Mauro Borges Lemos y Fernanda De Negri. 2006a. Impact of R\&D incentive program on the performance and technological efforts of Brazilian industrial firms. Documento de trabajo OVE 14/06. Washington: BID.

De Negri Joáo A., Mauro Borges Lemos y Fernanda De Negri. 2006b. The impact of university enterprise incentive program on the performance and technological efforts of Brazilian industrial firms. Documento de trabajo ove 13/06. Washington: BID. 
Duguet, Emmanuel. 2004. Are R\&D subsidies a substitute or a complement to privately funded R\&D? Evidence from France using propensity score methods for non-experimental data. Revue d'Économie Politique 114 (2): 245-274.

Georghiou, Luke, Bart Clarysse, Geert Steurs, Valentijn Bilsen y Jan Lacrosse. 2004. Making the difference. The evaluation of behavioural additionality of R\&D subsidies. IWT Studies, 48. Bélgica: IWT-Vlaanderen.

González, Xulia y Consuelo Pazó. 2008. Do public subsidies stimulate private R\&D spending? Research Policy 37: 271-389.

Hall, Bronwyn H. y Alessandro Maffiolli. 2008. Evaluating the impact of technology development funds in emerging economies: evidence from Latin America. European Journal of Development Research 20 (2): 172-198.

Hsu, Fang-Ming, Der-Juinn Horn y Chao-Chin Hsueh. 2009. The effect of governement-sponsored R\&D programmes on additionality in recipient firms in Taiwan. Technovation 29: 204-217.

Lööf, Hans y Almas Heshmati. 2005. The impact of public funds on private R\&D investment: new evidence from a firm level innovation study. Discussion papers 11862. Jokioinen Finland: MTT Agrifood Research Finland.

López, Andrés y Martin Rossi. 2010. Evaluación de impacto de un programa de financiamiento público a actividades de innovación en Uruguay-Programa Desarrollo Tecnológico. Monografía. Montevideo: Agencia Nacional de Investigación e Innovación.

López, Andrés, Ana M. Reynoso y Martin Rossi. 2010. Impact evaluation of a program of public funding of private innovation activities: an econometric study of FONTAR in Argentina. Documento de trabajo OVE 03/10. Washington: BID. 
López-Acevedo, Gladys y Hong W. Tan. 2010. Impact evaluation of SME programs in Latin America and the Caribbean. Washington: Banco Mundial.

Mercer-Blackman, Valerie. 2009. The impact of research and development tax incentives on Colombia's manufacturing sector: what difference do they make? Documento de trabajo wP/08/178. Washington: Fondo Monetario Internacional.

Mouqué, Daniel. 2012. What are counterfactual impact evaluations teaching us about enterprise and innovation support? Regional Focus 02. Brussels: DG Regional and Urban Policy.

Mungaray, Alejandro, Sergio López y Patricia Moctezuma. 2013. La adicionalidad de los fondos públicos en la innovación empresarial mexicana: el caso de Baja California, 2001-2010. Revista de La Educación Superior XLII (167): 41-62.

OECD. 2016. Main science and technology indicators, MSTI database, 2015-2 / enero. http://www.oecd.org/sti/msti.htm (8 de abril de 2016).

OECD. 2006. Government R\&D funding and company behaviour:measuring behavioural additionality. París: OECD.

OECD y Eurostat. 2005. Oslo manual. Guidelines for collecting and interpreting innovation data. París: OECD. http://www.oecd.org/sti/inno/oslomanualguidelinesforcollectingandinterpretinginnovationdata3rdedition.htm

Segura Mojica, Francisco Javier, Hugo Alejandro Borjas García y Adelita de Jesús Sifuentes Martínez. 2014. Evaluación del ambiente innovador en San Luis Potosí. región y sociedad Xxvi (61): 161-200.

Technopolis Group y Manchester Institute of Innovation Research. 2012. Evaluation of innovation activities. Guidance on methods and practices. Bruselas: European Commission, Directorate for Regional Policy. 
Vergara, Delia M. y Joost Heijs. 2013. El papel de las ayudas públicas para la innovación en las empresas del sector químico de México. Análisis micro. Ponencia presentada en el XV Congreso latino-iberoamericano de gestión tecnológica (ALTEC), Porto, Portugal.

Vila, Alonso M., Carlos F. Soto y Manuel G. González. 2010. Innovación, financiación pública y tamaño empresarial. Cuadernos de Gestión 10 (1): 75-88. 
